\title{
Corrections to the Banks-Casher relation with Wilson quarks
}

\section{CERN-PH-TH/2013-016}

\section{Silvia Necco}

CERN, Physics Departement, 1211 Geneva 23, Switzerland

\author{
Andrea Shindler*i \\ CERN, Physics Departement, 1211 Geneva 23, Switzerland \\ E-mail: andrea.shindler@cern.ch
}

\begin{abstract}
The Banks-Casher relation links the spectral density of the Dirac operator with the existence of a chiral condensate and spontaneous breaking of chiral symmetry. This relation receives corrections from a finite value of the quark mass, a finite space-time volume and, if evaluated on a discrete lattice, from the finite value of the lattice spacing $a$. We present a status report of a determination of these corrections for Wilson quarks.
\end{abstract}

The 7th International Workshop on Chiral Dynamics,

August 6 -10, 2012

Jefferson Lab, Newport News, Virginia, USA

\footnotetext{
* Speaker.

${ }^{\dagger}$ Heisenberg fellow
} 


\section{Introduction}

The Banks-Casher relation [1] relates the spectral density $\rho_{D}$ of the Hermitian Dirac operator $-i D$ with the chiral condensate $\Sigma$

$$
\rho_{D}(\gamma, m)=\frac{\Sigma}{\pi}\left[1+O\left(\{|\gamma|, m\} / \Lambda_{Q C D}\right)\right],
$$

where $\gamma_{k}$ are the eigenvalues of the massless operator and $m$ is the sea quark mass. In principle the Banks-Casher relation provides a tool to determine the chiral condensate [2] with a lattice QCD computation.

A more standard way to determine the chiral condensate with lattice QCD computations is to study the quark mass dependence of the pion mass and comparing it with the predictions of chiral perturbation theory $(\chi \mathrm{PT})$. Recent lattice calculations are performed close to the physical values of the quark masses (see ref. [3, 4] for recent reviews). To control accurately the light quark mass dependence of hadronic quantities it is important, if possible, to have independent determinations of leading order (LO) low energy constants (LECs), as for example the chiral condensate. In the plot in fig. 1 we show the light quark mass dependence of the squared pion mass computed by the European Twisted Mass Collaboration (ETMC) with $N_{f}=2$ dynamical light quarks [5]. Despite the rather good description of the lattice results with $\chi \mathrm{PT}$, it is obviously desirable being able to predict, in an independent way, the behaviour close to the chiral limit (red ellypse) to better constrain the light quark mass dependence. A constraint of the chiral fit will be beneficial for a more accurate determination of the next-to-leading order LECs and a better confidence on the chiral fits.

An example of independent determination of LO LECs is provided by lattice calculations in the so called epsilon-regime, where first estimates of the chiral condensate and decay constant are

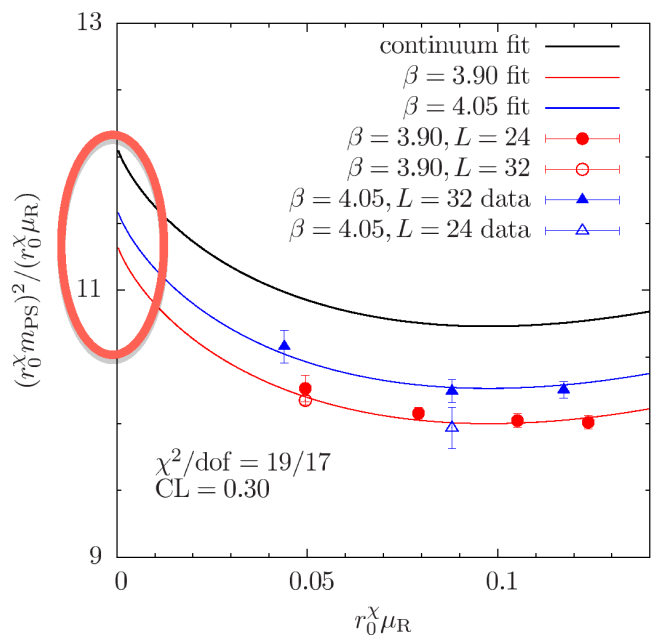

Figure 1: Light quark mass dependence of the ratio between the pseudoscalar meson mass squared and the renormalized light quark mass in units of the Sommer parameter $r_{0}$. rather encouraging [6,7]. Using Wilson-type fermions as a QCD discretization, calculations are affected by potentially large cutoff effects, thus it is important to have a theoretical analysis of quantities such as the spectral density, based on Wilson chiral perturbation theory (W $\chi \mathrm{PT})[10,11] .{ }^{1}$ These proceedings are a status report of an ongoing attempt to understand cutoff and finite size effects affecting the spectral density of the Wilson operator.

\section{Chiral condensate from the mode number}

With Wilson fermions it is advantageous to consider the Hermitean Wilson-Dirac operator $Q=$ $\gamma_{5} D_{m}$, where $D_{m}$ is the massive Wilson opearator. To compute the spectral density $\rho_{Q}$ in $\mathrm{W} \chi \mathrm{PT}$

\footnotetext{
${ }^{1}$ The analysis of two-point functions in the epsilon-regime within the framework of $\mathrm{W} \chi \mathrm{PT}$ has been discussed in refs. [12,13] and more recently extended in ref. [14].
} 

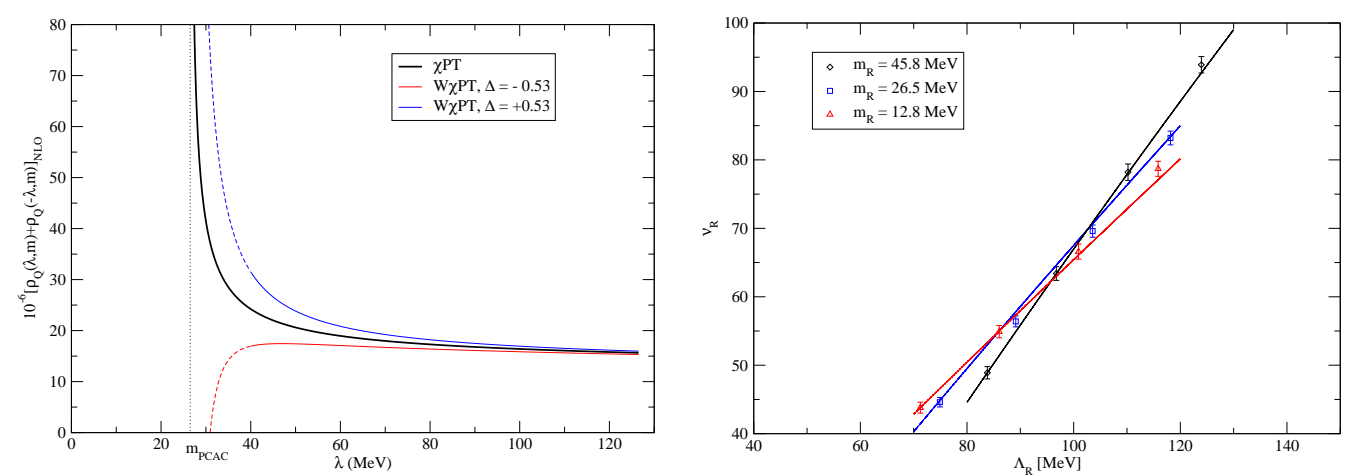

Figure 2: Left plot: the spectral density $\left[\rho_{Q}(\lambda, m)+\rho_{Q}(-\lambda, m)\right]_{N L O}$ in the infinite volume. We used the parameters $\Sigma=(275 \mathrm{MeV})^{3}, m_{\mathrm{PCAC}}=26.5 \mathrm{MeV}, F=90 \mathrm{MeV}, \bar{L}_{6}=5, \mu=139.6 \mathrm{MeV}$. The solid black line corresponds to the continuum $\chi$ PT prediction, while the red (blue) lines correspond to the lattice $\mathrm{W} \chi \mathrm{PT}$ prediction $\left(O(a)\right.$-improved) on eq. 2.3 with $\hat{a}^{2} W_{8}^{\prime}= \pm 5 \cdot 10^{6} \mathrm{MeV}^{4}$, corresponding to $\Delta=\mp 0.53$. Right plot: result of the global fit of the data published in [2] with our formula for the renormalised mode number (eqs. 2.4 and 2.3). The fit parameters are $\Sigma, \Delta$ and $\bar{L}_{6}$ and we obtain $\chi^{2} /$ dof $=0.91$.

one introduces a flavour doublet of valence fermions $\chi_{v}$ with a Wilson twisted mass action and twisted mass $\mu_{v}$. The spectral density is related to the discontinuity of the valence pseudoscalar condensate along the imaginary axis in the twised mass plane [15]

$$
\left.\operatorname{Disc}\left[\left\langle\bar{\chi}_{v} \gamma_{5} \tau^{3} \chi_{v}\right\rangle\right]\right|_{\mu_{v}=i \lambda}=2 i \pi\left[\rho_{Q}(\lambda, m)+\rho_{Q}(-\lambda, m)\right],
$$

thus the valence pseudoscalar condensate is a tool to compute the spectral density in $\mathrm{W} \chi \mathrm{PT}^{2}$. After matching the continuum Symanzik effective theory with the generalized effective chiral Lagrangian one needs to choose a proper power counting for the scales involved in the problem. The scales are the sea quark mass, $m$, the valence twisted mass $\mu_{v}$ (directly related to $\lambda$ ), the lattice spacing $a$ and the linear size of the space-time volume $L$. In the following we always consider the sea quark mass in the so-called $p$-regime. For the other scales we consider $m \sim \mu_{v} \sim a \sim 1 / L \sim O\left(p^{2}\right)$. The result of the calculation [16] in terms of the PCAC quark mass $m_{\mathrm{PCAC}}$ is given by

$$
\begin{aligned}
{\left[\rho_{Q}\left(\lambda, m_{\mathrm{PCAC}}\right)+\rho_{Q}\left(-\lambda, m_{\mathrm{PCAC}}\right)\right]_{N L O} } & =2\left[\rho_{Q}\left(\lambda, m_{\mathrm{PCAC}}\right)\right]_{N L O, \text { cont }} \\
& +\frac{2 \Sigma \lambda}{\pi \sqrt{\lambda^{2}-m_{\mathrm{PCAC}}^{2}}}\left[\frac{m_{\mathrm{PCAC}}^{2} \Delta}{\lambda^{2}-m_{\mathrm{PCAC}}^{2}}+\frac{16 \hat{a}}{F^{2}} W_{6}\right] .
\end{aligned}
$$

In these formula $\Delta=-\frac{16 \hat{a}}{F^{2}}\left(\frac{W_{8}}{2}+\frac{W_{10}}{4}+\frac{\hat{a} W_{8}^{\prime}}{M_{s s}^{2}}\right), M_{s s}$ is the pseudoscalar meson mass (made of two sea quarks), $\Sigma$ and $F$ are the LO LECs, $\hat{a}=2 W_{0} a$ and $W_{0}$ as the others $W$ s are the LECs parametrizing $\mathrm{O}(a)$ and $\mathrm{O}\left(a^{2}\right)$ effects. Details on the calculation and a discussion on the applicability of this formula can be found in refs. [16, 17].

Potentially even with Wilson fermions one can use the spectral density, or equivalently the renormalization group invariant (RGI) mode number [2]

$$
v(\Lambda, m)=V \int_{-\Lambda}^{\Lambda} d \lambda \rho_{Q}(\lambda, m)
$$

\footnotetext{
${ }^{2}$ We recall that in the continuum the two spectral densities are connected by the relation $\rho_{Q}(\lambda, m)=$ $\frac{\lambda}{\sqrt{\lambda^{2}-m^{2}}} \rho_{D}\left(\sqrt{\lambda^{2}-m^{2}}, m\right)$.
} 
to determine the chiral condensate. To test our formula we compared the resulting mode number (2.4) with the numerical data published in [2]. We have fixed $F=90 \mathrm{MeV}$ and the renormalisation scale $\mu=m_{\pi}$; we have performed a global fit at all the 3 masses available and all the values of $\Lambda_{R}$ with 3 fit parameters: $\Sigma, \Delta$ and $\bar{L}_{6}{ }^{3}$ From the global fit we obtain

$$
\Sigma^{1 / 3}=266(7) \mathrm{MeV}, \quad \Delta=-0.62(80), \quad \bar{L}_{6}=6(1) .
$$

The numerical data and our global fit are shown in the right plot of fig. 2. For $\Sigma$ we obtain a perfectly consistent result with Giusti and Lüscher [2] without performing any chiral extrapolation. We have also performed a fit fixing $F=80 \mathrm{MeV}$ obtaining compatible results within errors. Lattice determinations of the chiral condensate using the mode number can be found in $[2,8,9]$.

\section{Cutoff effects close to the threshold}

To improve the theoretical description of the spectral density of the Wilson operator for $\lambda \simeq m$ one needs to consider two important points. The first one is that finite size effects diverge for $\lambda \rightarrow m[16]$ and the second one is that the when $\lambda \simeq m$ the power counting for the valence quark masses need to be reconsidered, i.e. the treatment of the cutoff effects in a perturbative fashion might not be adequate.

To overcome this difficulties we opt for the following power counting

$$
m \sim O\left(p^{2}\right), \quad m_{P}=\sqrt{m_{v}^{2}+\mu_{v}^{2}} \sim O\left(p^{4}\right), \quad 1 / L, 1 / T \sim O(p), \quad a \sim O\left(p^{3}\right),
$$

that implies cutoff effects affecting the spectral density at NLO order. The framework is the socalled mixed Chiral Effective Theory [18], where some masses obey the p-regime counting, and others are in the epsilon-regime. As an intermediate step of our calculation we introduce a $\theta$-term solely in the mass-term of the action as follows

$$
\mathscr{L}_{2}=\frac{F^{2}}{4} \operatorname{Tr}\left[\partial_{\mu} U \partial_{\mu} U^{\dagger}\right]-\frac{\Sigma}{2} \operatorname{Tr}\left[U_{\theta}^{\dagger} U(x)^{\dagger} \mathscr{M}+\mathscr{M}^{\dagger} U(x) U_{\theta}\right]-\frac{\hat{a} F^{2}}{4} \operatorname{Tr}\left[U+U^{\dagger}\right],
$$

where the mass matrix in the replica formalism [19] is

$$
\mathscr{M}=\mathscr{M}^{\dagger}=\operatorname{diag}(\underbrace{m, \ldots, m}_{N_{s}}, \underbrace{m_{v}+i \mu_{v} \tau^{3}, \ldots, m_{v}+i \mu_{v} \tau^{3}}_{N_{r}}), \quad U_{\theta}=\operatorname{diag}\left(e^{\frac{i \theta}{N_{s}} \mathbb{1}_{s}}, \mathbb{1}_{r}\right) .
$$

While in the continuum it is not important how the $\theta$-term is introduced in the parametrization of the $U$-field $[18,20]$, adding a $\theta$-term in the sea sector only, becomes relevant at finite lattice spacing. With this choice, even with a $\theta$-term in the action, we can reabsorb the leading $\mathrm{O}(a)$ cutoff effects in a redifinition of the quark mass, because with our choice of power counting (3.1), in the sea sector the leading $\mathrm{O}(a)$ effects appear at NNLO, i.e. the sea quarks are effectively in the continuum up to higher order corrections. With this particular choice of power counting and parametrization of the $\theta$-term we can achieve, as in the continuum $[18,20]$, a factorization of the partition function for the zero and non-zero modes. The periodicity in $\theta$ of the chiral Lagrangian allows us to write the partition function in standard fashion

$$
\mathscr{Z}(\theta)=\sum_{v=-\infty}^{v=+\infty} e^{-i v \theta} \mathscr{Z}_{v}, \quad \mathscr{Z}_{v}=\frac{1}{2 \pi} \int_{0}^{2 \pi} d \theta e^{i v \theta} \mathscr{Z}(\theta) .
$$

\footnotetext{
${ }^{3}$ In this proceedings with $\Sigma$ we denote its value renormalised in the $\overline{M S}$ scheme at a scale of $2 \mathrm{GeV}$.
} 
By performing an exact integration over the constant field $\theta$ one obtains

$$
\mathscr{Z}_{v} \propto e^{\frac{-N_{s} 2^{2}}{22 s}} \int_{U\left(N_{r}\right)} d \bar{U}_{0}\left(\operatorname{det} \bar{U}_{0}\right)^{v} \mathrm{e}^{\frac{m_{v} \Sigma V}{2}} \operatorname{Tr}\left[\bar{U}_{0}^{\dagger}+\bar{U}_{0}\right], \quad z_{s}=m V \Sigma,
$$

from which one observes that the distribution of $v$ is Gaussian and it is controlled by the sea quarks which are in the $p$-regime. The computation of the spectral density is now a computation at fixed $v$, i.e. $\rho=\sum_{v} \rho_{v} \frac{\mathscr{Z}_{V}}{\mathscr{Z}}$. The sum over $v$ can be done because we know the weight factor $\frac{\mathscr{Z}_{V}}{\mathscr{Z}}$ (cfr. eq. 3.5). We expand the pseudo Nambu-Goldstone field $U(x)$ around the ground state of the theory $U_{V}$

$$
U_{V}=\operatorname{diag}(\underbrace{1}_{N_{s}}, \underbrace{e^{i \tau^{3} \omega_{0}}}_{N_{r}}), \quad \sin \omega_{0}=\frac{\mu_{v}}{m_{P}}, \quad \cos \omega_{0}=\frac{m_{v}}{m_{P}},
$$

and with this parametrization, the mass term in the chiral Lagrangian becomes like in the untwisted case, with a degenerate polar mass $m_{P}$ in the valence (replicated) sector.

We compute the pseudoscalar valence condensate (cf. eq. (2.1)) in the chiral effective theory,

$$
\left\langle P_{v}^{3}\right\rangle=\left.\frac{1}{V}\left\langle\frac{\partial}{\partial J} \int d^{4} x \mathscr{L}(x)\right\rangle\right|_{J=0},
$$

where now the source term has the following form

$$
\mathscr{M} \rightarrow \mathscr{M}_{J}=\mathscr{M}+J \hat{\tau}^{3}, \quad \mathscr{M}^{\dagger} \rightarrow \mathscr{M}_{J}^{\dagger}=\mathscr{M}^{\dagger}-J \hat{\tau}^{3}, \quad \hat{\tau}^{3}=\operatorname{diag}(\underbrace{0}_{N_{s}} \underbrace{\underbrace{\tau^{3}}_{2}, 0, \ldots, 0)}_{N_{r}} .
$$

The final result of the calculation can be written as

$$
\begin{aligned}
\left\langle P_{v}^{3}\right\rangle & =\frac{\Sigma_{\text {eff }}}{2} F_{1}+8 B \hat{a} W_{6} F_{2}+2 B \hat{a} W_{8} F_{3} \\
& +\frac{\Sigma}{2}\left[4 B \hat{a} W_{6} N_{s} m V F_{2}+\hat{a}^{2} V W_{6}^{\prime}\left(F_{4}+4 N_{s} F_{2}\right)+\hat{a}^{2} V W_{7}^{\prime} F_{5}+\hat{a}^{2} V W_{8}^{\prime} F_{6}\right],
\end{aligned}
$$

where $F_{i=1 . .6}$ are integrals over zero modes and $\Sigma_{\text {eff }}$ is an effective chiral condensate which includes NLO corrections from the sea quarks and the lattice spacing

$$
\Sigma_{\text {eff }}=\left(\Sigma_{\text {eff }}\right)_{\text {cont }}+16 \Sigma \frac{\hat{a} W_{6}}{F^{2}} .
$$

The explicit formula for $\left(\Sigma_{\text {eff }}\right)_{\text {cont }}$ can be found in [21]. In fig. (3) we show the impact of the NLO corrections to the chiral condensate [21]. One observes that in the typical range of quark masses for dynamical simulations the relative corrections induced by the presence of sea quarks can reach $30 \%$. This is a warning in case one would like to extract the chiral condensate from fits of mode num-

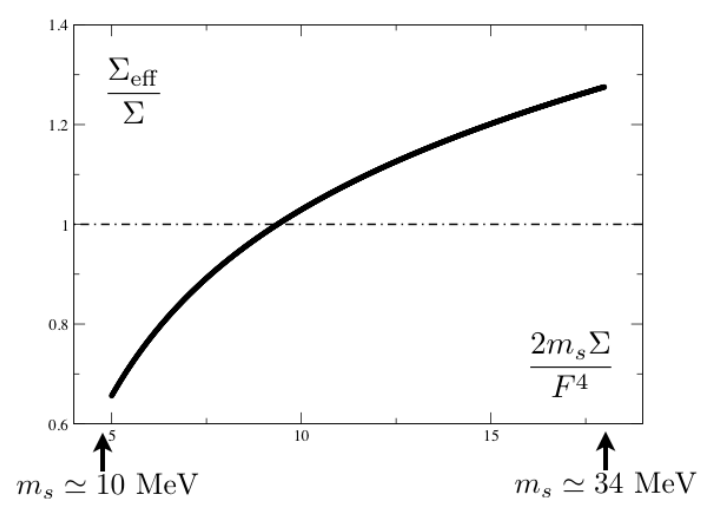

Figure 3: Significance of the NLO corrections for $\Sigma_{\text {eff }} / \Sigma$ in a typical range of sea quark masses for dynamical lattice QCD computations.

bers where the effect of the sea quarks have been neglected. To compute the zero-modes integrals 
we switch to the supersymmetric formulation. All integrals at fixed $v$ can be computed by derivating the graded $S U(2 \mid 2)$ partition function $[22,23]$ with respect to appropriately chosen sources. The integrals have been computed and cross-checked. The final step to determine the formula for the spectral density is the calculation of the discontinuity along the imaginary axis in the twisted valence mass plane (cfr. (2.1)). The analysis of the final result is in progress. We conclude observing that the effects of the sea quarks are twofold. They change the absolute normalization by introducing an $m$ dependence in $\Sigma_{\text {eff }}$ and they control the distribution of $v$, which, we remark, stays Gaussian only because the sea quarks are in the $p$-regime.

\section{References}

[1] T. Banks and A. Casher, Nucl.Phys. B169 (1980) 103.

[2] L. Giusti and M. Luscher, JHEP 03 (2009) 013, 0812.3638.

[3] C. Jung, PoS LAT2009 (2009) 002, 1001.0941.

[4] H. Wittig, PoS LATTICE2011 (2011) 025, 1201.4774.

[5] ETM Collaboration, R. Baron et al., JHEP 1008 (2010) 097, 0911.5061.

[6] K. Jansen, A. Nube and A. Shindler, PoS LATTICE2008 (2008) 083, 0810.0300.

[7] A. Hasenfratz, R. Hoffmann and S. Schaefer, Phys.Rev. D78 (2008) 054511, 0806.4586.

[8] JLQCD and TWQCD collaborations, H. Fukaya et al., Phys.Rev. D83 (2011) 074501, 1012.4052.

[9] K. Cichy et al., PoS LATTICE2011 (2011) 102, 1111.3322.

[10] S.R. Sharpe and J. Singleton, Robert L., Phys.Rev. D58 (1998) 074501, hep-lat/9804028.

[11] O. Bar, G. Rupak and N. Shoresh, Phys.Rev. D70 (2004) 034508, hep-lat/0306021.

[12] A. Shindler, Phys. Lett. B672 (2009) 82, 0812.2251.

[13] O. Bar, S. Necco and S. Schaefer, JHEP 03 (2009) 006, 0812.2403.

[14] G. Akemann and F. Pucci, (2012), 1211.3980.

[15] S.R. Sharpe, Phys. Rev. D74 (2006) 014512, hep-lat/0606002.

[16] S. Necco and A. Shindler, JHEP 04 (2011) 031, 1101.1778.

[17] S. Necco and A. Shindler, PoS LATTICE2011 (2011) 250, 1108.1950.

[18] F. Bernardoni and P. Hernandez, JHEP 10 (2007) 033, 0707.3887.

[19] P.H. Damgaard and K. Splittorff, Phys. Rev. D62 (2000) 054509, hep-lat/0003017.

[20] F. Bernardoni et al., JHEP 10 (2008) 008, 0808.1986.

[21] F. Bernardoni et al., Phys.Rev. D83 (2011) 054503, 1008.1870.

[22] Y.V. Fyodorov and G. Akemann, JETP Lett. 77 (2003) 438, cond-mat/0210647.

[23] K. Splittorff and J. Verbaarschot, Phys.Rev.Lett. 90 (2003) 041601, cond-mat/0209594. 